\title{
A New Physically-Based Simulation Framework for Modelling Flow-Like Landslides
}

\author{
Xilin Xia ${ }^{\mathrm{a}}$, Runqiu Huang ${ }^{\mathrm{b}}$, Qiuhua Liang ${ }^{\mathrm{a}}$, Bin $\mathrm{Yu}^{\mathrm{b}}$ \\ ${ }^{a}$ School of Civil Engineering and Geosciences, Newcastle University, Newcastle upon Tyne, UK \\ ${ }^{\mathrm{b} S t a t e}$ Key Laboratory of Geohazards Prevention and Environment Protection, \\ Chengdu University of Technology, Chengdu 610059, China
}

\begin{abstract}
Flow-like landslides are one type of the most catastrophic natural hazards that often cause significant loss of life and property. Numerical modelling has become a powerful tool to facilitate better risk management of this type of natural hazards. In the past decades, a number of models have been developed to simulate flow-like landslides. Among them, the depth-integrated models based on continuum theory have gained wide-spreading popularity. However, these models are generally developed either on the locally curved coordinate system that prevents the direct use of geographic information system (GIS) data or by not taking into full account of the vertical acceleration on steep slopes that may lead to loss of accuracy. These limitations hinder the wider application of these models in simulating field-scale landslides with complex topography. This paper presents a new depthintegrated model for more robust simulation of flow-like landslides. The model is developed on a fixed global Cartesian coordinate system to enable the direct use of the widely available GIS data. To gain more favourable accuracy, the new model also takes into account the effect of the vertical acceleration. The improved performance of this new model is validated against an experimental test case.
\end{abstract}

Keywords: natural hazards, flow-like landslides, GIS, depth-integrated model, risk assessment

\section{Introduction}

Flow-like landslides are one type of the most catastrophic natural hazards that often lead to significant loss of life and property (Jakob et al 2005). Typically initiated on steep slopes, flowlike landslides may travel at high velocity for a long distance. Prediction of the run-out distance and velocity of such an event is essential to facilitate risk assessment and enable the design of appropriate protection measures to mitigate the possible human and economic losses.

Current approaches to predicting the run-out distance and velocity of flow-like landslides may be generally classified into two categories: empirical-statistic methods and dynamical methods. The empirical-statistic approaches are commonly easy to implement and use, but their applications are typically limited and should only be applied to the cases with predominating conditions similar to those based on which they are developed. Dynamic models are developed based on the physical laws of mass and momentum conservation and therefore can be used to more reliably represent the physics of landslides. They can be further categorised into lumped mass models and continuum mechanics based models. For example, the lumped mass model developed by Hutchinson (1986) idealised the flow mass into a single slab for simplicity, which is not able to simulate internal deformation and the movement of flow front.

*Corresponding Author: Qiuhua Liang, email: qiuhua.liang@ncl.ac.uk; Tel: +44 (191) 208-6413 
Compared with the lumped mass models, the continuum mechanics based models are much more versatile for different applications. Savage and Hutter (1989) firstly developed a model for granular avalanches on rough inclining slopes. In their model, the internal stress and the resistant force between flow mass and bed are derived according to the Mohr-Columb assumption, i.e. the shear stress is only dependent on the normal stress; the resulting governing equations are depth-averaged along the vertical axis. In the model developed later by Hungr (1995), alternatives to the Mohr-Columb assumption were adopted to represent the resistant force between the flow mass and the channel bed while the form of internal force remained the same as that used by Savage and Hutter (1989). In this model, the flow mass was divided into a finite number of columns and the relative motion between these columns was controlled by the interacting forces. However, the effect of pore water pressure is not fully taken into account in these models.

To better simulate the effect of pore water pressure, Iverson (1997) adapted the SavageHutter model (1989) to consider the pore water pressure as a constant during a simulation, which was originally developed for one-dimensional problems. Later, Iverson and Denlinger (2001) introduced a two-dimensional and fully coupled model for mass movement. In their model, a formula based on the Biot consolidation theory was used to allow an ad-hoc estimation of the rapidly varying pore water pressure during landslide simulations. The model solved the depth-integrated governing equations using a finite volume scheme incorporating with an approximate Riemann solver to ensure shockcapturing capability. Lagrangian models have also been reported for landslide simulations in the last two decades, which include, for example, the Lagrangian finite element model introduced by Chen and Lee (2000) and the smoothed particle hydrodynamics (SPH) models by Pastor et al (2009) and McDougall and Hungr (2004). All of the aforementioned continuum mechanics based models adopt the Savege-Hutter formulation (Savage and Hutter 1989) to calculate the internal stress, assuming that the direction of principal stress can be prescribed. This assumption restricts the flexibility of these models in applications. In order to overcome this, Denlinger and Iverson (2004) introduced a finite element model in which the internal stress was locally calculated, removing the necessity of prescribing the direction of principal stress.

The above physically based flow-like landslide models are usually based on depthintegrated governing equations obtained through integrating the full mathematical model along the vertical axis, considering the fact that flow-like landslides are typically featured with vertical scale much smaller than the horizontal extent. This is similar to deriving the shallow water equations from the Navier-Stokes equations and is a common practice in computational fluid dynamics to optimise the solution accuracy and computational efficiency. However, because the bed slopes on which landslides occur are usually steep, the vertical movement of the flow mass cannot be neglected and hence the hydrostatic assumption may no longer be valid when deriving the governing equations through depthintegration. Aiming to resolve this issue, researchers (Savage and Hutter 1989, Hungr 1995, Denlinger and Iverson 2001) developed their models using a curved coordinate system with the $x-o-y$ plane attached to the earth surface, ensuring that the $z$ axis was always perpendicular to the Earth's surface. However, when applying the depth-averaged models for field-scale simulations, the earth surface elevation, usually described by a Digital Elevation Model (DEM), is needed to quantify the gravity driving force. However, the DEM datasets are typically stored on a rasterbased format (or a fixed Cartesian coordinate system); a landslide model based on a curved coordinate system cannot be directly integrated with geographic information system (GIS) tools to process the DEM datasets; complex coordinate transformation between the Cartesian coordinate system and curvilinear coordinate system is therefore mandatory, inevitably leading to smoothened topographic features and loss of accuracy.

In this work, a new model based on the Savage-Hutter formulation is developed on a fixed Cartesian coordinate system, with the 
vertical axis defined along the direction of gravity, to enable direct use of GIS datasets. Meanwhile, in order not to compromise the solution accuracy, a modified formula depending on bed slope is derived for gravity acceleration to incorporate the effect of vertical movement into the internal stress and resistant force.

\section{Mathematical Model}

The formulation introduced in this section is based on a fixed right-hand Cartesian coordinate system with the vertical axis parallel to the direction of gravity, as shown in Fig.1.



Fig. 1 The coordinate system.

In this work, the flow mass is treated as a single phase, assuming a constant density. The full mathematical governing equations can be derived from the mass and momentum conservation laws:

$\nabla \mathbf{v}=0$

$\rho\left(\frac{\partial \mathbf{v}}{\partial t}+\mathbf{v} \cdot \nabla \mathbf{v}\right)+=-\nabla \mathbf{T}+\rho \mathbf{g}$

where $v$ is the velocity of the flow mass, $\rho$ is the density, $T$ is the internal forces and $g$ is the body force. For landslides, the horizontal extent is usually much larger than the vertical scale, a depth-integrated formulation can be subsequently derived through integrating Eq. (1) and (2) along the vertical axis, similar to deriving the depthaveraged shallow water equations (Vreugdenhil 1994). For one-dimensional problems, the final depth-averaged governing equations are given as: $\frac{\partial h}{\partial t}+\frac{\partial h \bar{v}_{x}}{\partial x}=0$

$\rho\left(\frac{\partial h \bar{v}_{x}}{\partial t}+\frac{\partial h \bar{v}_{x}^{2}}{\partial x}\right)=-\frac{\partial \bar{T}_{x x} h}{\partial x}+\left.T_{x x}\right|_{b e d} \frac{\partial z}{\partial x}-\left.T_{\mathrm{zx}}\right|_{b e d}$

$\left.T_{z z}\right|_{b e d}=\rho g h+\rho h \frac{d \bar{v}_{z}}{d t}$

where $h$ is the flow depth, $\bar{v}_{x}$ is the depthaveraged velocity, $\bar{T}_{x x}, \bar{T}_{z x}$ and $\bar{T}_{z z}$ are the stresses with the subscript 'bed' denoting the stresses acting on the bed, and $g$ is the gravity acceleration. Because flow-like landslides usually occur on steep slopes, the effect of vertical acceleration on the stress term in (5) must be considered and a total vertical acceleration can be defined as:

$g^{\prime}=g+\frac{d \bar{v}_{z}}{d t}$

As suggested by other researchers (e.g. Denlinger and Iverson 2001), the stresses in (4) are proportional to the vertical normal stress:

$\bar{T}_{x x}=\frac{1}{2} k T_{z z}=\frac{1}{2} k \rho g^{\prime} h$

$\left.T_{x x}\right|_{\text {bed }}=\left.k T_{z z}\right|_{\text {bed }}=k \rho g^{\prime} h$

$\left.T_{\mathrm{zx}}\right|_{\text {bed }}=\left.k^{\prime} T_{z z}\right|_{\text {bed }}=k^{\prime} \rho g^{\prime} h$

As seen from (7) - (9), the vertical acceleration may have significant effects on the internal stresses in (4). However, the total vertical acceleration $g^{\prime}$ is not straightforward to be determined. Therefore, an approximate approach is proposed based on the stresses acting on the bed. From Cauchy's theorem, $\left.T_{x x}\right|_{b e d} \partial z / \partial x-\left.T_{z x}\right|_{\text {bed }}$ is equivalent to $T_{n x} / \cos \alpha$ while $T_{n x}$ may be further represented by $\sigma_{n}$ and $\tau_{n}$, i.e. $T_{n x}=\sigma_{n} \sin \alpha+\tau_{n} \cos \alpha$, as shown in Fig. 2. Similarly, $\left.T_{z z}\right|_{\text {bed }}+\left.T_{x z}\right|_{\text {bed }}$ can be evaluated by $T_{n z} / \cos \alpha$, where $T_{n z}=\sigma_{n} \cos \alpha+\tau_{n}$ $\sin \alpha$. In order to evaluate $\left.T_{z z}\right|_{\text {bed }}$, an approximation is made so that $\left.T_{x z}\right|_{\text {bed }}=\tau_{n} \sin \alpha$. 
The resulting formula for $\left.T_{x x}\right|_{b e d} \partial z / \partial x-\left.T_{z x}\right|_{b e d}$ and $\bar{T}_{x x}$ are:

$\left.T_{x x}\right|_{b e d} \frac{\partial z}{\partial x}-\left.T_{z x}\right|_{b e d}=\sigma_{n} \sin \alpha / \cos \alpha+\tau_{n}$

$\bar{T}_{\mathrm{xx}}=\left.\frac{1}{2} k T_{z z}\right|_{b e d}=\frac{1}{2} k \sigma_{n}$



Fig. 2 The stresses on a triangular element

It is straightforward to obtain $\sigma_{n}$ and $\tau_{n}$ if a uniform layer assumption is made, so that the depth of the flow mass is of a constant depth, i.e. $d h / d x=0$, which can be justified in most of the field-scale flow-like landslides. Under this assumption, $\sigma_{n}$ and $\tau_{n}$ are given as:

$\sigma_{n}=\rho g h \cos ^{2} \alpha$

$\tau_{n}=\rho g h \cos ^{2} \alpha \tan \varphi_{b e d} \cdot \operatorname{sgn}\left(v_{x}\right)$

where $\varphi_{b e d}$ is the friction angle of the bed, $\alpha$ is the slope angle, as illustrated in Fig. 3.



Fig. 3 The uniform slab assumption.
After substituting (10) - (13) into (4), the momentum equation becomes:

$$
\begin{aligned}
& \frac{\partial h \bar{v}_{x}}{\partial t}+\frac{\partial\left(h \bar{v}_{x}^{2}+\beta k g h^{2} / 2\right)}{\partial x} \\
& =-\cos ^{2} \alpha g h\left(\frac{\partial z}{\partial x}+\tan \varphi_{b e d} \cdot \operatorname{sgn}\left(v_{x}\right)\right)
\end{aligned}
$$

where $z$ is the bed elevation that can be directly obtained from a DEM. $\cos ^{2} \alpha$ may be calculated by

$$
\cos ^{2} \alpha=\frac{1}{1+(\partial z / \partial x)^{2}}
$$

The coefficient $k$ may be quantified using the formula introduced by Savage and Hutter (1989), based on the Mohr-Columb assumption:

$$
k=k_{\text {act pass }}=2 \frac{1 \mp\left[1-\cos ^{2} \varphi_{\text {int }}\left(1+\tan ^{2} \varphi_{\text {bed }}\right)\right]^{1 / 2}}{\cos ^{2} \varphi_{\text {int }}}-1
$$

where - and + denote the active and passive stresses for diverging flows and converging flows, and $\varphi_{\text {int }}$ and $\varphi_{\text {bed }}$ are the friction angles of the flow mass material and bed material, respectively.

In summary, the depth-averaged governing equations are now given by:

$\frac{\partial \mathbf{q}}{\partial t}+\frac{\partial \mathbf{f}}{\partial x}=\mathbf{s}$
$\mathbf{q}=\left[\begin{array}{c}h \\ h \bar{v}_{x}\end{array}\right]$,

$\mathbf{f}=\left[\begin{array}{c}h \bar{v}_{x} \\ h \bar{v}_{x}^{2}+k \beta g h^{2} / 2\end{array}\right]$,

$$
\begin{aligned}
& \mathbf{s}=\left[\begin{array}{c}
0 \\
-\beta g h\left(\partial z / \partial x+\tan \varphi_{b} \cdot \operatorname{sgn}\left(v_{x}\right)\right)
\end{array}\right] \\
& k=k_{\text {act pass }}=2 \frac{1 \mp\left[1-\cos ^{2} \varphi_{\text {int }}\left(1+\tan ^{2} \varphi_{\text {bed }}\right)\right]^{1 / 2}}{\cos ^{2} \varphi_{\text {int }}}-1
\end{aligned}
$$

$\beta=\frac{1}{1+(\partial z / \partial x)^{2}}$ 
The effect of the vertical acceleration has now been taken into account in (17) - (20). Except for the bed friction and internal stress coefficient $k$, the only difference between the above governing equations for flow-like landslides and the shallow water equations is that the gravity acceleration $g$ has been corrected by $\beta g$. Therefore, the numerical schemes developed for the shallow water equations can be directly applied to solve equations (17) - (20).

\section{Numerical Model}

In this paper, the governing equations (17) - (20) for flow-like landslides are solved using a finite volume Godunov-type scheme, with a HartenLax-van Leer (HLL) approximate Riemann solver (Harten et al 1983) implemented to evaluate the fluxes across cell interfaces. After applying Green's theorem on the integral form of (17), the finite volume discretised formula becomes:

$$
\mathbf{q}_{i}^{n+1}=\mathbf{q}_{i}^{n}-\frac{\Delta t}{\Delta x}\left(\mathbf{f}_{i+1 / 2}-\mathbf{f}_{i-1 / 2}\right)+\Delta t \mathbf{s}_{i}
$$

where $i$ denotes the cell index, $\Delta t$ and $\Delta x$ are the time step and cell size, respectively. According to the solution structure of the HLL approximate Riemann solver as shown in Fig. 4, the fluxes across a cell interface are calculated by

$$
\mathbf{f}=\left\{\begin{array}{cc}
\mathbf{f}_{L} & 0 \leq S_{L} \\
\mathbf{f}_{*} & S_{L}<0<S_{R} \\
\mathbf{f}_{R} & S_{R} \leq 0
\end{array}\right.
$$

where $\mathbf{f}_{\mathrm{L}}=\mathbf{f}\left(\mathbf{q}_{\mathrm{L}}\right)$ and $\mathbf{f}_{\mathrm{R}}=\mathbf{f}\left(\mathbf{q}_{\mathrm{R}}\right)$ are calculated from the Riemann states $\mathbf{q}_{\mathrm{L}}$ and $\mathbf{q}_{\mathrm{R}}$ defining a local Riemann problem. The fluxes in the middle region $\mathbf{f} *$ are calculated from the HLL formula (Harten et al 1983):

$$
\mathbf{f}_{*}=\frac{S_{R} \mathbf{f}_{L}-S_{L} \mathbf{f}_{R}+S_{L} S_{R}\left(\mathbf{q}_{R}-\mathbf{q}_{L}\right)}{S_{R}-S_{L}}
$$

The wave speeds are determined from

$$
S_{\mathrm{L}}=\left\{\begin{array}{cc}
\bar{v}_{x, R}-2 \sqrt{k \beta g h_{R}} & h_{L}=0 \\
\min \left(\bar{v}_{x, L}-\sqrt{k \beta g h_{L}}, \bar{v}_{x, *}-\sqrt{k \beta g h_{*}}\right. & h_{L}>0
\end{array}\right.
$$

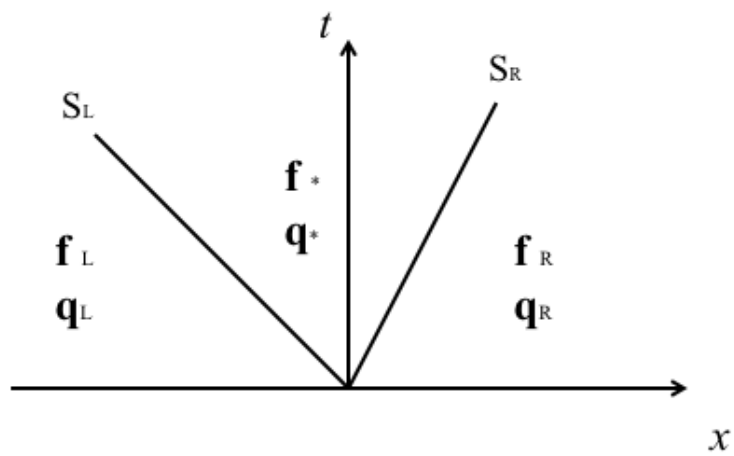

Fig. 4 The solution structure of the Harten-Lax-van Leer (HLL) approximate Riemann solver

$S_{\mathrm{R}}=\left\{\begin{array}{cc}\bar{v}_{x, L}-2 \sqrt{k \beta g h_{R}} & h_{R}=0 \\ \min \left(\bar{v}_{x, R}-\sqrt{k \beta g h_{R}}, \bar{v}_{x, *}-\sqrt{k \beta g h_{*}}\right. & h_{R}>0\end{array}\right.$

in which $\bar{v}_{x, *}$ and $h_{*}$ are calculated by

$$
\begin{aligned}
& \bar{v}_{x, *}=\frac{1}{2}\left(\bar{v}_{x, L}+\bar{v}_{x, R}\right)+\sqrt{k \beta g h_{L}}-\sqrt{k \beta g h_{R}} \\
& h_{*}=\frac{1}{g}\left[\frac{1}{2}\left(\sqrt{k \beta g h_{L}}+\sqrt{k \beta g h_{R}}\right)+\frac{1}{4}\left(\bar{v}_{x, L}-\bar{v}_{x, R}\right)\right]^{2}
\end{aligned}
$$

When calculating the Riemann fluxes $k$ and $\beta$ using the following formula, the averaged values of $k=\left(k_{\mathrm{L}}+k_{\mathrm{R}}\right) / 2$ and $\beta=\left(\beta_{\mathrm{L}}+\beta_{\mathrm{L}}\right) / 2$ are taken to maintain stability:

$k_{i}=\left\{\begin{array}{cl}k_{a c t} & \bar{v}_{x, i+1}-\bar{v}_{x, i-1}>0 \\ 1 & \bar{v}_{x, i+1}-\bar{v}_{x, i-1}=0 \\ k_{\text {pass }} & \bar{v}_{x, i+1}-\bar{v}_{x, i-1}<0\end{array}\right.$

$\beta_{i}=\frac{1}{1+(\partial z / \partial x)_{i}{ }^{2}}$

with

$\left(\frac{\partial z}{\partial x}\right)_{i}=\frac{z_{i+1}-z_{i-1}}{2 \Delta x}$

The source term is calculated by

$\mathbf{s}_{i}=\left[\begin{array}{c}0 \\ -\beta g h_{i}\left((\partial z / \partial x)_{i}+\tan \varphi_{b} \cdot \operatorname{sgn}\left(\bar{v}_{x, i}\right)\right)\end{array}\right]$

and 


\section{Model Validation and Results}

In this section, the new depth-integrated model for flow-like landslides is validated by applying it to an experimental case reported in Hutter et al (1995). As shown in Fig. 5, $1730 \mathrm{~cm}^{3}$ of glass beads were placed at the end of a $10 \mathrm{~cm}$ wide chute behind a gate. The chute consisted of an inclined segment and a level segment. The sloping part had an angle of $40^{\circ}$ and a length of $144 \mathrm{~cm}$. The friction angles of the flow mass and the bed material were $30^{\circ}$ and $22^{\circ}$, respectively. After the gate was suddenly opened at $t=0$, the glass beads rapidly flowed down the slope and deposited at the level segment of the chute. During the experiment, the dynamic flow process was recorded using a high-speed camera. The video files were post-processed to provide a series of front and rear positions of the moving mass.

In the simulation, the friction of the side walls are also considered and calculated by

$$
f_{w}=-0.5 \beta k g h^{2} \operatorname{sgn}\left(\bar{v}_{x}\right) \tan \varphi_{b}
$$

$f_{w}$ is included in (31) as part of the source terms. Fig. 6 compares the front and rear positions of the flow mass recorded during the experiments and predicted by the current model. The numerical prediction agrees well with the experimental measurements; small discrepancy exists but it is normal for this type of simulations. This confirms the acceptable solution accuracy of the current model and implies its potential for further development and practical applications.

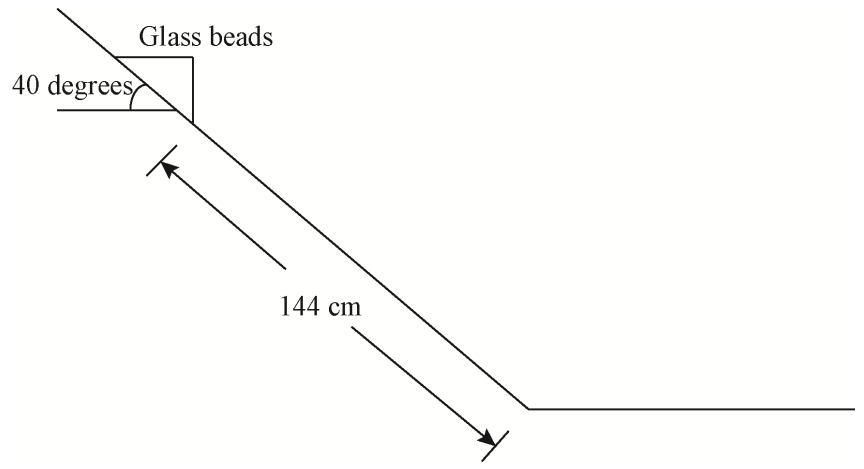

Fig. 5 The experiment setup (Hutter et al 1995).

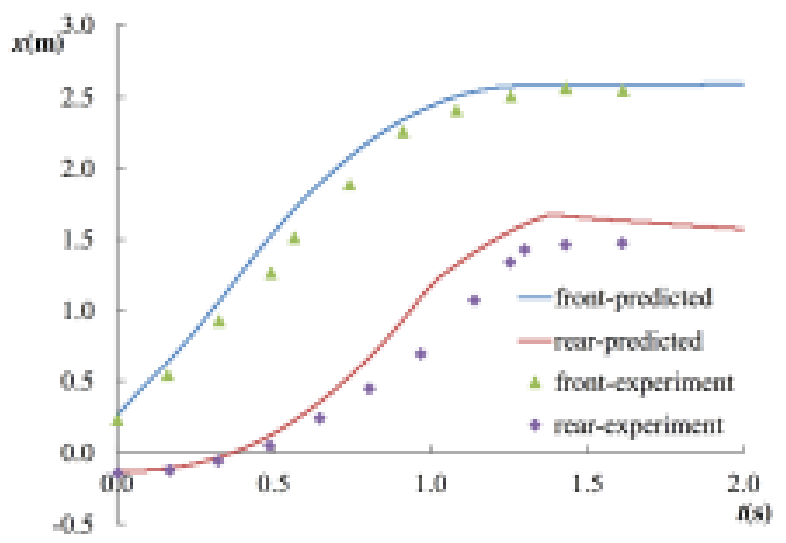

Fig. 6 Predicted and measured front and rear positions of the moving mass. Experiment data obtained from Hutter et al (1995)

\section{Conclusions and Future Work}

In this work, a new depth-integrated model for flow-like landslides has been proposed. This model is derived on a fixed Cartesian coordinate system to facilitate direct use of GIS data in engineering applications. In order to take into account the effect of vertical acceleration for simulations on steep slopes, the gravity acceleration in the depth-integrated governing equations has been corrected by including a coefficient relevant to the slope angle. The resulting equations are solved using a finite volume Godunov-type scheme. The resulting model has been validated against an experimental test case with satisfactory results obtained.

The current model provides a useful tool to predict the run-out distance and velocity of a flow-like landslide event and to assess possible risks. Currently developed for one-dimensional problems, the modelling framework may be easily extended to two-dimensional for field-scale applications involving more complex landslide dynamics over steep and rough domains in the future.

\section{Acknowledgement}

This work is partly funded by the open fund (SKLGP2015K002) from the State Key Laboratory of Geohazards Prevention and Environment Protection, Chengdu University of Technology, China. 


\section{References}

Chen, H. and C.F. Lee, 2000. Numerical simulation of debris flows. Canadian Geotechnical Journal, 37: 146 - 160.

Iverson, R.M and R. P. Denlinger, 2001. Flow of variably fluidized granular masses across three-dimensional terrain: 1 . Columb mixture theory. Journal of Geophysical Research, 106 (B1): 537 - 552.

Denlinger, R.P. and R.M. Iverson, 2001. Flow of variably fluidized granular masses across three-dimensional terrain: 2. Numerical Predictions and Experimental Tests. Journal of Geophysical Research, 106 (B1): 553 - 566.

Delinger, R.P. and R.M. Iverson, 2004. Granular avalanches across irregular three-dimensional terrain: 1. Theory and Computation. Journal of Geophysical Research, 109 (F1): F01014. doi:10.1029/2003JF000085

Harten, A., P. Lax and B. Leer, 1983. On upstream differencing and godunov-type schemes for hyperbolic conservation laws. SIAM Review, 25 (1). Society for Industrial and Applied Mathematics. pp: 35 - 61 . doi: $10.1137 / 1025002$

Hungr, O., 1995. A model for the runout analysis of rapid flow slides, debris flows, and avalanches. Canadian Geotechnical Journal, 32: 610 - 623 .

Hutchinson, J.N., 1986. A sliding-consolidation model for flow slides. Canadian Geotechnical Journal, 23: 115 - 126.
Hutter, K., T. Koch, C. Pluüss and S.B. Savage, 1995. The dynamics of avalanches of granular materials from initiation to runout. Part II. Experiments. Acta Mechanica, 165: 127 - 165. Iverson, R.M., 1997. Hydraulic modeling of unsteady debris-flow surges with solid-fluid interactions. In: Proceedings of First International Conference on Debris-Flow Hazards Mitigation. pp: 550 - 560. San Francisco: ASCE.

Jakob, M., O. Hungr and D.M. Jakob, 2005. Debris-dlow hazards and related phenomena. Springer.

Mcdougall, S. and O. Hungr, 2004. A model for the analysis of rapid landslide motion across three-dimensional terrain. Canadian Geotechnical Journal, 1097: 1084 - 1097. doi:10.1139/T04-052

Pastor, M., B. Haddad, G. Sorbino, S. Cuomo and V. Drempetic, 2009. A depth-integrated, coupled SPH model for flow-like landslides and related phenomena. International Journal for Numerical and Analytical Methods in Geomechanics, 33(2). John Wiley \& Sons, Ltd. pp: 143 - 172. doi:10.1002/nag.705

Savage, S.B. and K. Hutter, 1989. The motion of a finite mass of granular material down a rough incline. Journal of Fluid Mechanics, 199: 177 - 215.

Vreugdenhil, C.B., 1994. Numerical methods for shallow-water flow. Springer. 